\title{
Gamma-ray imaging with coaxial HPGe detector
}

T. Niedermayr, K. Vetter, L. Mihailescu, G. J. Schmid, D. Beckedahl, J. Kammeraad, J. Blair

May 3, 2005

Nuclear Instrumentations and Methods in Physics Research Section A 
This document was prepared as an account of work sponsored by an agency of the United States Government. Neither the United States Government nor the University of California nor any of their employees, makes any warranty, express or implied, or assumes any legal liability or responsibility for the accuracy, completeness, or usefulness of any information, apparatus, product, or process disclosed, or represents that its use would not infringe privately owned rights. Reference herein to any specific commercial product, process, or service by trade name, trademark, manufacturer, or otherwise, does not necessarily constitute or imply its endorsement, recommendation, or favoring by the United States Government or the University of California. The views and opinions of authors expressed herein do not necessarily state or reflect those of the United States Government or the University of California, and shall not be used for advertising or product endorsement purposes. 


\title{
Gamma-ray imaging with a coaxial HPGe detector
}

\author{
T. Niedermayr ${ }^{1}$, K. Vetter ${ }^{1}$, L. Mihailescu ${ }^{1}$, G. J. Schmid ${ }^{1}$, D. Beckedahl ${ }^{1}$, J. Blair ${ }^{2}$, J. \\ Kammeraad $^{1}$ \\ ${ }^{1}$ Lawrence Livermore National Laboratory, Livermore CA 94550, USA \\ ${ }^{2}$ Bechtel Nevada, North Las Vegas, NV 892310, USA
}

\begin{abstract}
:
We report on the first experimental demonstration of Compton imaging of gamma rays with a single coaxial high-purity germanium (HPGe) detector. This imaging capability is realized by two-dimensional segmentation of the outside contact in combination with digital pulse-shape analysis, which enables to image gamma rays in $4 \square$ without employing a collimator. We are able to demonstrate the ability to image the $662 \mathrm{keV}$ gamma ray from a ${ }^{137} \mathrm{Cs}$ source with preliminary event selection with an angular accuracy of 5 degree with an relative efficiency of $0.2 \%$. In addition to the $4 \square$ imaging capability, such a system is characterized by its excellent energy resolution and can be implemented in any size possible for Ge detectors to achieve high efficiency.
\end{abstract}

PACS: 29.40.G, 89.20. + a, 07.85

Keywords: Gamma-ray imaging, segmented HPGe detector, Compton camera 


\section{Introduction}

The ability to image and characterize known as well as unknown gamma-ray sources is finding a variety of applications in biomedical research and nuclear medicine, astrophysics, national security, such as nuclear nonproliferation, stockpile stewardship, nuclear waste monitoring and, most recently, nuclear counterterrorism. While gamma-ray imaging is an established tool in nuclear medicine or astrophysics, only recently the impact of gamma-ray imaging for nuclear security applications has been recognized. Here, the goal is to provide improved capabilities to detect, localize, and characterize nuclear materials. One of the outstanding challenges in homeland security is the detection and identification of nuclear threats in the midst of a sea of non-threat objects, which consists of legitimate radioactive objects commonly found in commerce and environment. In addition to the detection of nuclear materials which can be achieved by simple counting instruments, it is crucial to obtain as much information from this material as possible to mitigate the primarily nuisance alarms. This can be achieved by identifying the radioisotope via its characteristic gamma-ray decay, and by imaging it, e.g. by measuring the location and the shape of sources. In addition to the ability to localize and image gamma-ray source, gamma-ray imaging can potentially increase the sensitivity in finding such sources, particularly in complex and changing backgrounds due to the ability to improve signal-to-background. In particular, collimator-less Compton imaging systems enable to measure signals and background simultaneously and therefore potentially provide the biggest gain in signal-to-background. Gamma-ray imagers based 
on position-sensitive semi-conductor detectors such as high-purity Ge (HPGe) provide excellent imaging and spectroscopic characteristics and therefore fulfill both important requirements in national security.

Well established means of imaging consist of a mechanical and passive collimator such as parallel-hole or pinhole systems in front of a position sensitive gamma-ray detectors [1]. These systems suffer from the trade-off between efficiency and resolution. More advanced collimator- or aperture based instruments consists of modulated apertures either in space - coded aperture - or time - rotation modulation aperture - which have limited capabilities in high activity and complex backgrounds. The ideal gamma-ray imager, a gamma-ray lens, is very difficult to realize due to the small angle of total reflection. Multilayer, diffractive optics system have built which are able to focus gamma-rays up to $160 \mathrm{keV}$, however, these systems are characterized by a large focal length and small field-of-view [2]. An alternate way to image gamma-rays without the use of a collimator is Compton imaging. Recent advances in the two-dimensional segmentation of semiconductor detectors along with signal processing allow us now to build efficient and high-resolution Compton imaging systems.

We report on one possible implementation, which consists of a two-dimensionally segmented, coaxial HPGe detector. Other approaches consist of planar configurations made of a variety of materials such as $\mathrm{Si}, \mathrm{Ge}$, or $\mathrm{CdZnTe}$, either in double-sided strip or pixelated geometry $[3,4,5]$. The advantage of a coaxial HPGe detector is the large volume of a single detector that can be manufactured, which translates into high efficiencies for 
gamma-ray energies up to several $\mathrm{MeV}$. In addition, intrinsic properties of high-purity $\mathrm{Ge}$ enable excellent energy resolution and signal-noise ratios. The atomic number of $Z=32$ represents an acceptable compromise between efficiency which requires high $\mathrm{Z}$ and Compton imaging sensitivity which requires low $\mathrm{Z}$. The latter is due to the fact that for Compton imaging at least one Compton scatter process is required before the gamma ray is absorbed via the photo-electrical effect, and the two first interaction have to be sufficiently far apart that one is able to separate them and one is able to deduce the scattering angle with finite accuracy.

In the following, we will briefly introduce the concept of Compton imaging in $\mathrm{Ge}$ detectors. In chapter 3, we will introduce the 40-fold segmented coaxial HPGe detector, which was built by ORTEC and used for our experiments. In chapter 4 we discuss pulseshape analysis procedures to deduce three-dimensional positions for individual gammaray interactions. Chapter 5 finally illustrates measurements, which demonstrate Compton imaging in such a detector and discusses the impact of improvements in the pulse-shape analysis of multiple interaction which occur close to each other.

\section{Compton imaging with a HPGe detector}

As first published by Todd for nuclear medicine [6] and Schönfelder for astrophysics [7], the concept of Compton imaging relies on the Compton scattering process and the relationship between the scattering angle $\square$, the energy of the incident gamma ray $E_{\square}$ and the energy of the first interaction $E_{1}$ : 


$$
\cos (\square)=1+\frac{511}{E_{\square}} \square \frac{511}{E_{\square}^{\prime}} ; \quad E_{\square}^{\prime}=E_{\square} \square E_{1}
$$

As illustrated in fig.1, the scattering angle describes a cone whose symmetry axis is defined by the line connecting the positions of the first two interactions. The projection of those cones on a sphere will overlap at the source location when many events are imaged or backprojected. Without measuring the direction of the Compton electron the incident angle of the gamma ray can only be determined to be on a cone surface. Since in Ge, the range of electrons is typically below $1 \mathrm{~mm}$ (for instance, a $1 \mathrm{MeV}$ gamma ray generates an electron of about $500 \mathrm{keV}$ which has a range of about $0.5 \mathrm{~mm}$ in $\mathrm{Ge}$ ) it is very difficult to measure the scattering angle of the electron, particularly considering the complex slowing-down process of electrons. Only in low-Z or low-density detectors such as gases at about 1 atm electron vertices could be measured [8]. However, the efficiency to induce a gamma-ray interaction at all in these materials is extremely low.

The angular resolution that can be obtained in Compton imaging depends on the uncertainties in the determination of the positions of the interactions and the deposited energies. The error in the positions affects the cone axis direction while the error in the energies affects the angle _ of the cone. The position and energy resolution can in principle be improved for better angular resolution but the electron on which the photon Compton scatters carries momentum, which is inherently unknown and will limit the best angular resolution attainable [9]. In order to improve image quality, a large number of image reconstruction algorithms have been developed [10-13]. The iterative List Mode Maximum Likelihood (LMML) algorithm which is well suited for low statistics data can improve angular resolution as shown later. 
In Ge, Compton scattering is the dominant interaction process between $150 \mathrm{keV}$ and about $8 \mathrm{MeV}$. However, at low energies, e.g. below $300 \mathrm{keV}$, the energy of the scattered gamma ray is so low that in most of the cases it is absorbed by the photoelectrical effect within $2 \mathrm{~mm}$ which makes it very difficult to identify two interactions and to separate them. But even if they can be separated, the uncertainty in the position measurement, particularly if based on pulse-shape analysis at low energies, will limit the angular resolution significantly. This is one reason which explains why previous attempts to use coaxial Ge detectors as Compton imagers failed [14]. For gamma-ray energies of $500 \mathrm{keV}$ a higher angular resolution of a few degrees should be readily achievable. For gamma-ray energies above $1 \mathrm{MeV}$ the angular resolution should be even better and ultimately limited by the range of the Compton scattered electron.

If only two interactions are involved in the event, a simple energy-ratio consideration can identify which of the two interactions is most likely the first one [15]. For higher gamma-ray energies, i.e. $500 \mathrm{keV}$, two and more Compton scattering processes become more likely before the gamma ray is absorbed. To obtain the proper scattering sequence, gamma-ray tracking algorithms have to be employed [16-18]. These gamma-ray tracking algorithms not only provide the most likely scattering sequence but also provide the likelihood of a full-energy deposition or an escape event, which means, that the gamma ray was not fully absorbed in the detector but escaped before. In this way they can improve the spectroscopic response of the detector, e.g. by increasing the peakto-total ratio [16]. 
In summary, the full volume imager combines high efficiency and high energy resolution but is limited by the separation ability of events which scatter close by, which reduces the ultimately achievable angular resolution to a few degrees.

\section{Coaxial HPGe prototype imager}

In the approach presented here, three-dimensional positions and energies of gammaray interactions are obtained by pulse-shape analysis in a two-dimensionally segmented, coaxial HPGe detector, which was manufactured by ORTEC. The closed-end crystal is of n-type with the segmented B-contact outside and the unsegmented Li contact inside. The impurity concentration was provided by the manufacturer to be $5 \times 10^{-9} \mathrm{~cm}^{-3}$ in the front and $10 \times 10^{-9} \mathrm{~cm}^{-3}$ in the back of the crystal. The crystal's diameter is $5 \mathrm{~cm}$, the overall length $8 \mathrm{~cm}$. It is segmented 40 -fold on the outside cylindrical surface only to simplify pulse shape analysis: 8 longitudinal segments separated by $\Delta_{-}=45^{\circ}$ (labeled A through H) and 5 transverse segments separated by $\Delta \mathrm{z}=1 \mathrm{~cm}$ (labeled 1 through 5), as illustrated

in fig. 2. The B-contact at the front $2 \mathrm{~cm}$, which contains the complex, pseudo-planar electrical fields and the rear $1 \mathrm{~cm}$ were left without segmentation. The crystal is oriented in such a way as to have the major crystallographic axes aligned with the segmentation lines. This results in a similar charge collection with respect to the azimuthal angle and avoids transfer of charge carriers from one electrode to another during the collection process. This simplifies the simulation of the charge transport and the signal shapes. Custom preamplifiers built around warm FET's are mounted on a circular motherboard 
close to the detector to reduce input capacitance. A picture of the detector aluminum housing with the preamplifier visible is also shown in fig. 2. A digital signal acquisition system manufactured by Struck Innovative Systems (SIS) is used to read out the 40 segment channels as well as the front, and the central contact with a $100 \mathrm{MHz}$ sampling rate and 12 bit ADC's. The data from the 8-channel digitizer boards are read through a VME-PCI interface and processed and analyzed on a PC. The typical energy resolution obtained is $0.9 \mathrm{keV}$ and $1.9 \mathrm{keV}$ at gamma-ray energies of $60 \mathrm{keV}$ and $1332 \mathrm{keV}$, respectively. The energy resolution of the central channel was degraded to about $2 \mathrm{keV}$ at $60 \mathrm{keV}$ due to the leakage current on the rear side of the Ge detector. The degradation could have been prevented by isolating the rear und non-segmented side by electrically segmenting the inner contact.

\section{Position determination through pulse shape analysis}

In order to determine the 3-D positions of the interaction sites of a gamma-ray inside a detector, a detailed understanding of the resulting pulse shapes is necessary. The shape of the signal generated on the charge collecting electrode as well as the shape of the induced signals produced on the neighboring electrodes is a unique signature of the 3D position of the interaction.

When gamma-ray photons interact in Ge, they can produce a recoil electron or a photoelectron at each interaction site. These electrons will lose about a third of their energy by generating electron-hole pairs and two thirds by exciting lattice vibrations. The 
charge cloud, which is left along the track of the primary electron has a size on the order of the stopping distance. In the following, the charge cloud is considered to be generated instantaneously and its extent is neglected in the simulations. The lateral diffusion of charge carriers perpendicular to the electrical field is neglected as well since the maximum range can be estimated to be smaller than $100 \square \mathrm{m}$, which is small compared to the overall dimensions of the segments considered in the detector.

A signal is produced by the charge cloud by inducing an image charge of opposite polarity at the electrodes. The charge is accelerated under the influence of the applied electric field and reaches an equilibrium velocity, which is considered here to be reached instantaneously for simplification. This charge drift in the detector induces a change in the image charge at the electrodes. If the charge is produced far away from the destination electrode, the induced charge is distributed over several electrodes. As the charge moves closer to its destination electrode, the charge is increased on the collecting electrode and the induced charge on the neighboring electrodes decreases until the charge finally reaches the destination electrodes. Thus a net charge is only measured on the destination electrode while the neighboring electrodes have transient signals, which vanish when the charge is collected.

The first step in calculating signals which can be observed at the segments is the determination of the path of the charge carriers inside the detector volume for a given position of the interaction. The charge migrates under the influence of the applied electric field, which depends on the detector geometry, applied voltage, intrinsic space-charge 
density _ and carrier mobility _. The electric field is calculated for the detector geometry by solving the Poisson equation using the finite-element method program MAXWELL 3D:

$$
\square \square(r)=\frac{\square \square(z)}{\square}
$$

An electric potential is thus obtained for the three dimensional detector volume with a Cartesian grid size of $1 \mathrm{~mm}$. The electric field can then be calculated:

$$
\dot{E}^{\prime}(r)=\square \square(\stackrel{r}{r})
$$

The trajectory of the electrons and holes can be calculated using the velocity:

$$
\stackrel{r}{v}(\stackrel{r}{r})=\square \cdot E \cdot(r)
$$

The electric field is interpolated between grid points. A time interval of $10 \mathrm{~ns}$ is used in generating the charge carrier trajectories, small enough to prevent any drift velocities discontinuities. The charge carrier mobility depends on the temperature, electric field, the angle between the drift velocity and crystal orientation and additionally on the angle between the electric field and crystal orientation $[19,20]$. The latter dependency is not taken into account here due to the difficulty in a closed description for the hole mobility, which implies that charge carriers are only allowed to move along the electrical fields.

The next step in calculating the signals generated at the electrodes is to calculate the weighted potential $[21,22]$. The Laplace equation is solved with voltage applied only on the collecting electrode and all the other contacts grounded. By calculating the electric field for each grid point and electrode and using the previously calculated charge carrier trajectories, the induced charge can be obtained with: 


$$
\square Q_{i, j}=\frac{q_{0} \dot{E}_{j}\left(r_{i}\right) \square r_{i}^{r}}{V_{0}}
$$

where $\mathrm{q}_{0}$ is the charge deposited and $\mathrm{V}_{0}$ the applied voltage. Thus, for each 1835 positions on the $1 \mathrm{~mm}$ grid in each segment, the signals generated at each electrode have been calculated. These signals are used to determine the positions of interactions, e.g. by least-square minimization procedures.

\section{Experimental imaging with the coaxial detector}

\section{5.a. signal mapping measurements}

In order to validate and adjust the simulated signals, measurements resulting from interactions with defined positions were carried out. In order to restrict the interactions to Compton scatters at $90^{\circ}$ along a line in the HPGe coaxial imager, a collimated source was used and a HPGe coaxial "catcher" detector was operated in coincidence. The experimental setup is shown in fig. 3 . A ${ }^{137} \mathrm{Cs}$ point source is collimated behind two Heavimet bricks separated by $1.5 \mathrm{~mm}$, thus forming a plane parallel to the detector transverse direction z. A coaxial HPGe "catcher" detector is placed behind two heavimet bricks separated by $1.5 \mathrm{~mm}$, defining a plane of sight at $90^{\circ}$ in respect to the source illumination plane. A line of possible interactions is thus defined by the intersection of these two planes, parallel to the imager $\mathrm{z}$ axis, with a diameter of approximately $2 \mathrm{~mm}$. In order to restrict events to ${ }^{137} \mathrm{Cs} 662 \mathrm{keV}$ photons scattering at $90^{\circ}$ in the imager and subsequently absorbed in the "catcher" detector, the "catcher" detector was operated in coincidence with the imager and energy gates were set on both detectors. Thus only 
events which deposit $374 \mathrm{keV}$ in the imager and $278 \mathrm{keV}$ in the "catcher" were recorded. The "catcher" detector was shielded with lead in order to reduce false or random coincidences. Monte Carlo simulations show that more than $90 \%$ of all coincidence events measured in this way are due to single interaction events in the imager. Both Heavimet collimators are mounted on translation stages in order to scan the imager in two dimensions, which was done on 12 different positions on a $3 \mathrm{~mm}$ grid, as shown in fig. 4 . Due to symmetry considerations, these 12 positions are sufficient to characterize the whole detector volume. The overall alignment was determined by matching intensity ratios of different segments which proved to be accurate to better than $1 \mathrm{~mm}$.

Out of the 5 illuminated segments F1...F5 (where F corresponds to the longitudinal position and 5 to the transverse position), only events in the middle three (F2...F4) were saved in order to be able to record the signal on the charge collecting electrode as well as the induced signals on all of the 8 neighboring electrodes. The measured energy correlation between the imager and "catcher" detectors is shown in fig. 5. The shape and width of the peak is determined by the Compton profile in Ge reflecting the electron momentum on which the photon Compton scatters.

Since the experimental setup only provides a line of interactions in the detector, the point of interaction has to be inferred by the amplitude of the signals. This is done by calibrating the signal position in accordance with the range of amplitudes. The advantage of this experimental procedure compared to reducing the interaction sites to a point is a large reduction in the number of necessary measurements and therefore measurement 
time. In fig. 6, signals from the charge collecting electrode and its 8 neighbors is shown for two different positions. The induced signals are crucial in order to determine the 3D position of the interaction. They can have either polarity, depending on the radius of the interaction which determines if electrons or holes will dominate in the signal. On one hand, this has the advantage of increasing the dynamic range of the signals thereby potentially increasing the position sensitivity. On the other hand the induced signals from the electrons and holes can cancel each other out for interactions in certain areas of the segment thus decreasing the sensitivity there. Since the position of the interaction in the measurements is not restricted to a point but to a line parallel to the longitudinal segmentation, a set of signals with different amplitudes on top and bottom and similar amplitudes left and right are recorded.

In order to validate the simulated signals, the calibration signals are treated as unknown data for which the positions must be determined. In this way the position resolution of the system can be determined since the position of the measured signals is known. The measured signals are fitted with simulated signals using a ${ }_{-}^{2}$ minimization procedure. The reconstructed positions for six calibration points are shown in fig. 7. Although there is excellent agreement between measured and calculated signals, inhomogeneous distributions of positions and deviations of up to $3 \mathrm{~mm}$ can be observed. The reconstructed positions are shifted towards segment boundaries, e.g. areas of higher sensitivity. These effects are due to the reduced sensitivity in the middle of the segments (regions furthest away from the electrode edges). 


\section{5.b. Compton imaging}

To demonstrate the imaging capabilities, measurements were carried out by illuminating the detector with a ${ }^{137} \mathrm{Cs}$ source from two meters away. Only events which deposit the full gamma-ray energy in the three middle layers were considered to ensure that all segments hit have a complete set of neighbor signals. In addition, the interactions were required to take place in two different segments separated by a distance large enough in order to be able to fit the measured signals and thus extract the energy and location of the events. In practice, this meant a separation of at least two segments between the active segments. In our experiment, $0.4 \%$ of all events collected fulfilled these conditions. This is in fair agreement with $0.8 \%$ expected by Monte-Carlo simulations performed with GEANT [23]. Fig. 8 shows the energy and position distributions of the high and low energy events. The first interaction, closer to the source, is the one with low energy, as expected for $662 \mathrm{keV}$ photons. With the positions and energies determined, it is possible to reconstruct an image with simple cone backprojection. The resulting image is shown on the left-hand side in fig.9. By applying a listmode maximum-likelihood algorithm [24], the image on the right of fig.9 is obtained after 10 iterations, resulting in an angular resolution of about 5 degree.

Monte-Carlo simulations mentioned above indicate that about $80 \%$ of all gamma rays emitted in the solid angle of the detector interact with the detector, which means that the overall efficiency for the implemented event selection, which resulted in an angular resolution of 5 degree is about $0.3 \%$. More sophisticated signal-decomposition methods, 
which enable the analysis of multiple interactions in one or adjacent segments it should be possible to increase the sensitivity to larger than $1 \%$. Assuming the position resolution of $3 \mathrm{~mm}$ as achieved so far the angular resolution will degrade to about 10 degree. However, if the position resolution can be improved to $1 \mathrm{~mm}$, the previous angular resolution of 5 degree can be achieved with an efficiency of $5 \%$

\section{Conclusion}

We have demonstrated Compton imaging in a two-dimensionally segmented, coaxial HPGe detector by using pulse-shape analysis to deduce the three-dimensional positions of interactions. With simple event-selection criteria, we obtain about 5 degree angular resolution at a energy of $662 \mathrm{keV}$ with an efficiency of $3 \times 10^{-3}$ in $4 \square$. Monte-Carlo simulations indicate that with more sophisticated signal-decomposition methods, which enable the analysis of multiple interactions in one or adjacent segments it should be possible to increase the sensitivity to larger than $1 \%$. Assuming the position resolution of $3 \mathrm{~mm}$ as achieved so far the angular resolution will degrade to about $10 \mathrm{deg}$. However, if the position resolution can be improved to $1 \mathrm{~mm}$, the angular resolution of $5 \mathrm{deg}$. can be achieved with an efficiency of 5\%.

With the excellent intrinsic properties for gamma-ray spectroscopy of a coaxial HPGe detector and now in addition, the imaging capability with a sensitivity of larger than $1 \%$ and a resolution of about 5 deg., such an instrument becomes attractive for a variety of applications, e.g. for nuclear materials monitoring or emergency response. 


\section{Acknowledgements}

We thank Pat Sangsingkeow from ORTEC for her support in the endeavor of making this detector work. Special thanks to Harold Yaver and Michael Maier from Lawrence

Berkeley National Laboratory for the hard work on the preamplifiers.

The work was performed under the auspices of the U.S. Department of Energy by University of California Lawrence Livermore National Laboratory under contract No. W7405-Eng-48.

\section{References}

[1] Physics in Nuclear Medicine, 2nd ed., J.A. Sorenson and M. Phelps; W. B. Saunders Company: Philadelphia, PA 1987

[2]D.L. Windt et al., App. Opt. 42 (2003) 2415

[3] Y. Du, Z. He, G.F. Knoll, D.K. Wehe, and W. Li, Nucl. Instr. and Meth. A457 (2001) 203

[4] B.F Phlips, et al., IEEE Trans. Nucl. Sci. 43 (1996) 1472

[5] K. Vetter, M. Burks and L. Mihailescu, Nucl. Instr. Meth. Phys. Res. A 525 (2004) 322

[6] R.W. Todd, J.M. Nightingale,and D.B. Everett, Nature 251 (1974) 132

[7] V. Schoenfelder et al., Nucl. Instr. and Meth. 107 (1973) 385

[8] R. Bellazzini et al., Nucl. Instr. and Meth. 535 (2004) 477

[9] D. Brusa et al., Nucl. Instr. and Meth. 379 (1996) 167

[10] L. Parra and H.H. Barrett, IEEE Trans. Nucl. Sci. NS-17 (1998) 228

[11] L.A. Shepp and Y. Vardi, IEEE Trans. Nucl. Sci. NS- 1 (1982) 113

[12] S.J. Wilderman et al., IEEE trans Nucl. Sci. NS-48 (2001) 111 
[13] R. Basko, G. L. Zeng and G. T. Gullberg, Physics in Medicine and Biology 43 (1998) 887

[14] G.J. Schmid, D. A. Beckedahl, J.J. Blair, J.E. Kammeraad, K. Vetter, A. Kuhn, Nucl. Instr. and Meth. A459 (2001) 565

[15] C.E. Lehner, Z. He, F. Zhang, IEEE Trans. Nucl. Sci. NS-51 (2004) 1618

[16] G.J. Schmid et al., Nucl. Instr. and Meth. A430 (1999) 69

[17] G.J. Schmid et al., IEEE Trans. Nucl. Sci. NS-44 (1997) 975

[18] J. van der Marel and B. Cederwall, Nucl. Instr. and Meth. A437 (1999) 538

[19] G. Ottaviani, C. Canali, A. Albergi Quaranta, IEEE Trans. Nucl. Sci. NS-22 (1975) 192

[20] L. Reggiani, C. Canali, F. Nava and G. Ottaviani, Phys. Rev. B 16 (1977) 2781

[21] S. Ramo, Proc. IRE 27 (1939) 584

[22] Radiation Detection and Measurement, $3^{\text {rd }}$ ed. , G.F. Knoll, Wiley, New York, 2000

[23] GEANT3, Detector Description and Simulation Tool, CERN, Geneva, 1993

[24] L. Mihailescu et al.., submitted to Nucl. Instr. and Meth. 


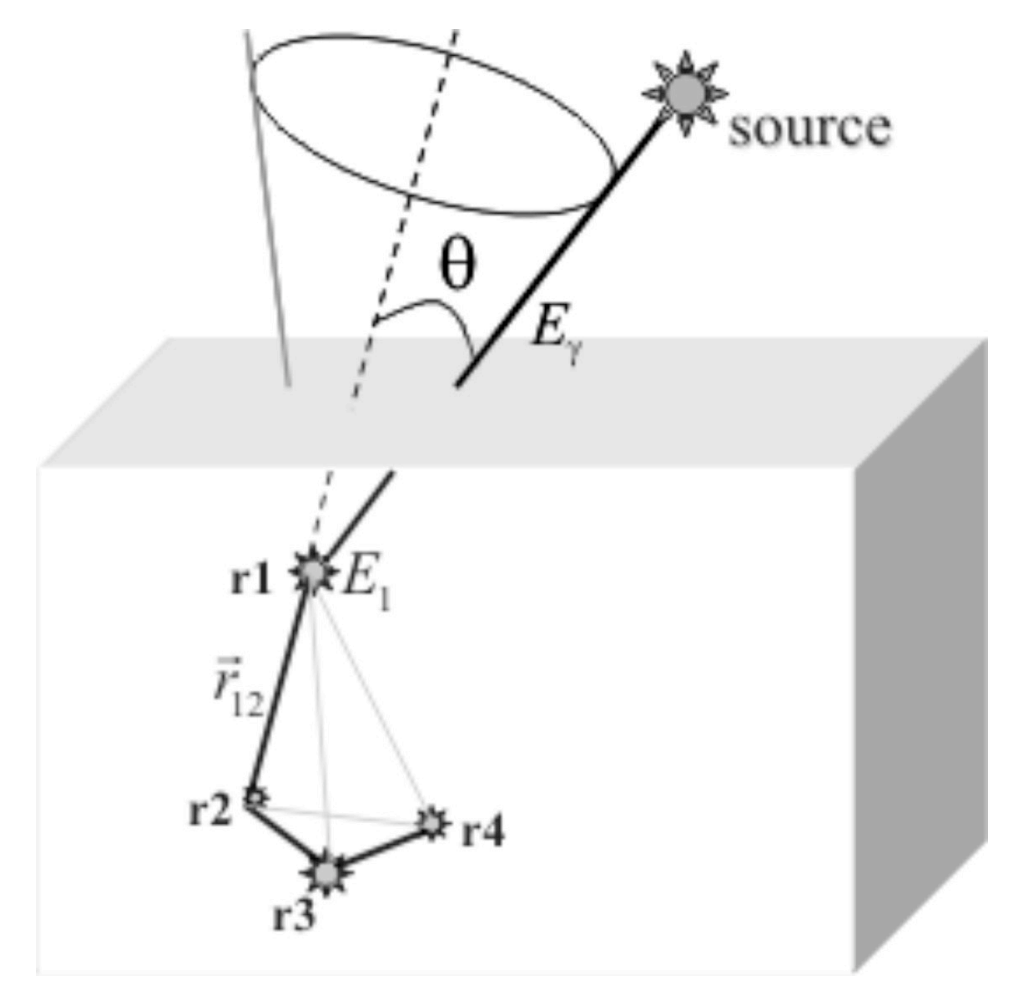

Fig. 1: Illustration of Compton imaging principle. The positions of the first two interactions define the symmetry axis of a cone whose opening angel is defined by the energy of the first interaction and the total gamma-ray energy.

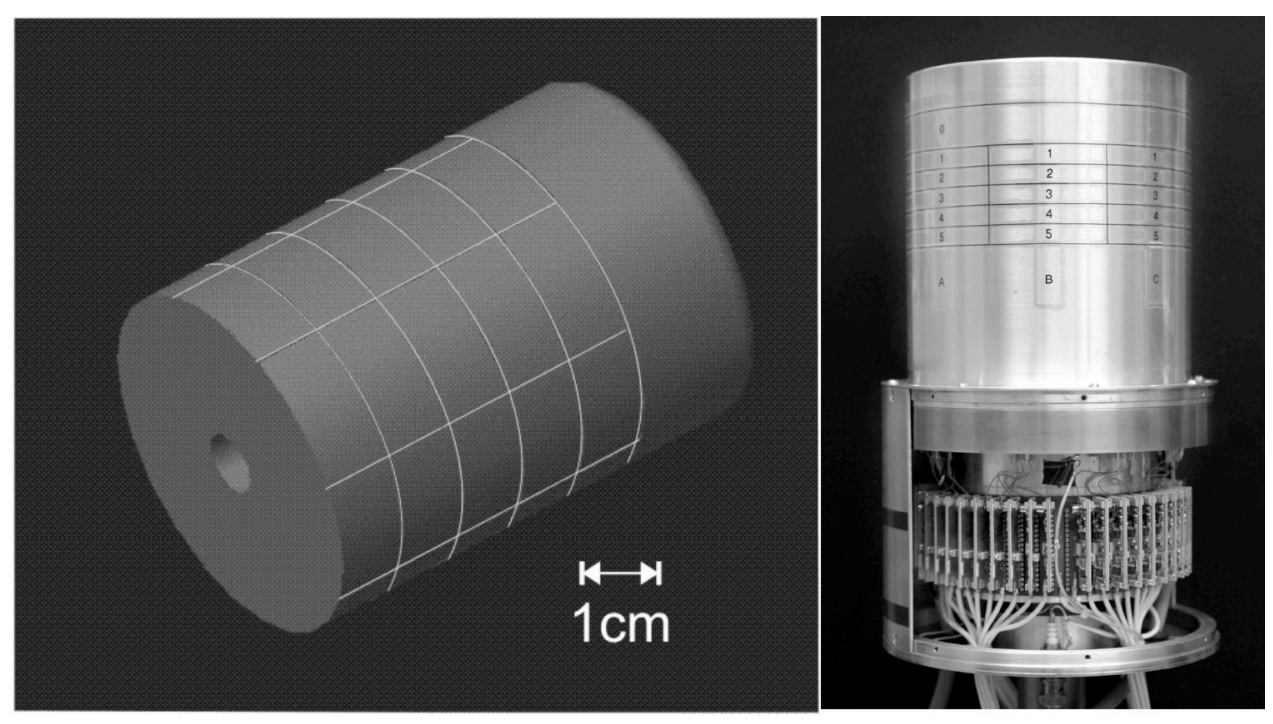

Fig. 2: 40-fold segmented, coaxial HPGe detector. The segmentation scheme is indicated on the left, the detector housing and the preamplifier arrangement can be seen on the right. 




Fig. 3: Coincidence setup used to map out signals within the detector. A $1 \mathrm{mCi}$

${ }^{137} \mathrm{Cs}$ source is located in a hevimet block with a slit opening of $1.5 \mathrm{~mm}$ to define a plane of interactions in the detector. At 90 deg., a second HPGe detector is mounted behind another hevimet absorber with a slit opening of $1.5 \mathrm{~mm}$. These slits define a line of possible interactions when a coincidence between the coaxial imager and the coaxial catcher detector is required.

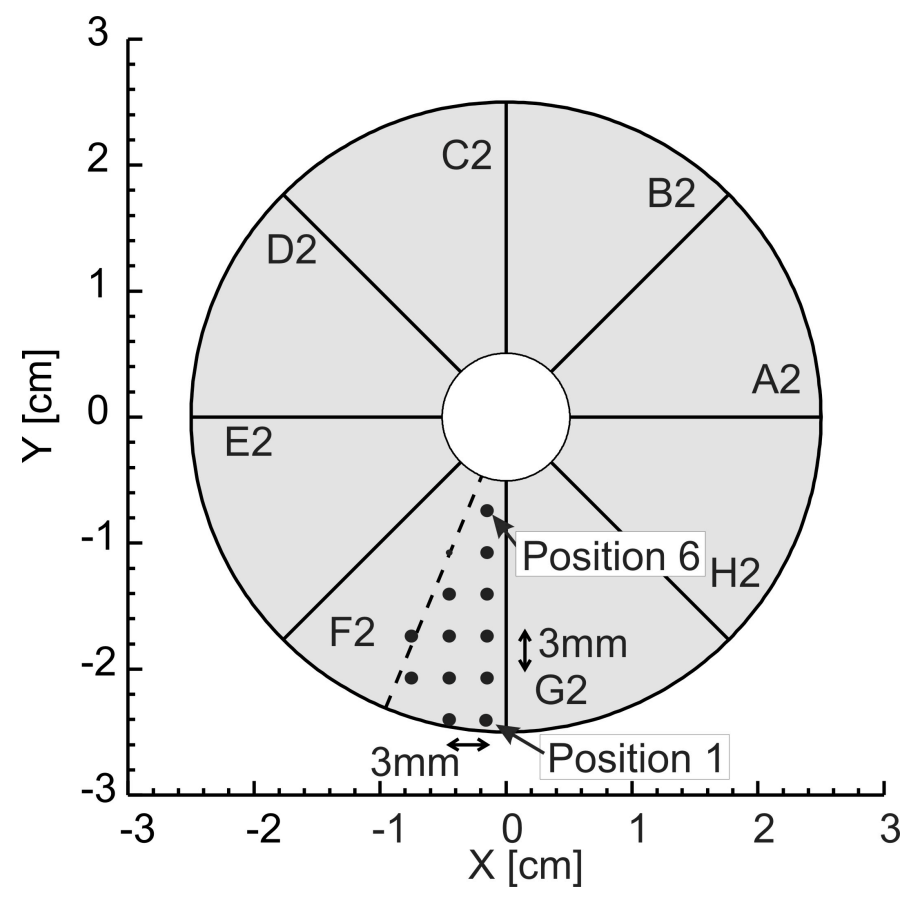

Fig. 4: $12 \mathrm{X}-\mathrm{Y}$ positions of collimator during coincidence measurements. Segments F1 to F5 were illuminated while only segments F2 to F4 were used in the trigger. 

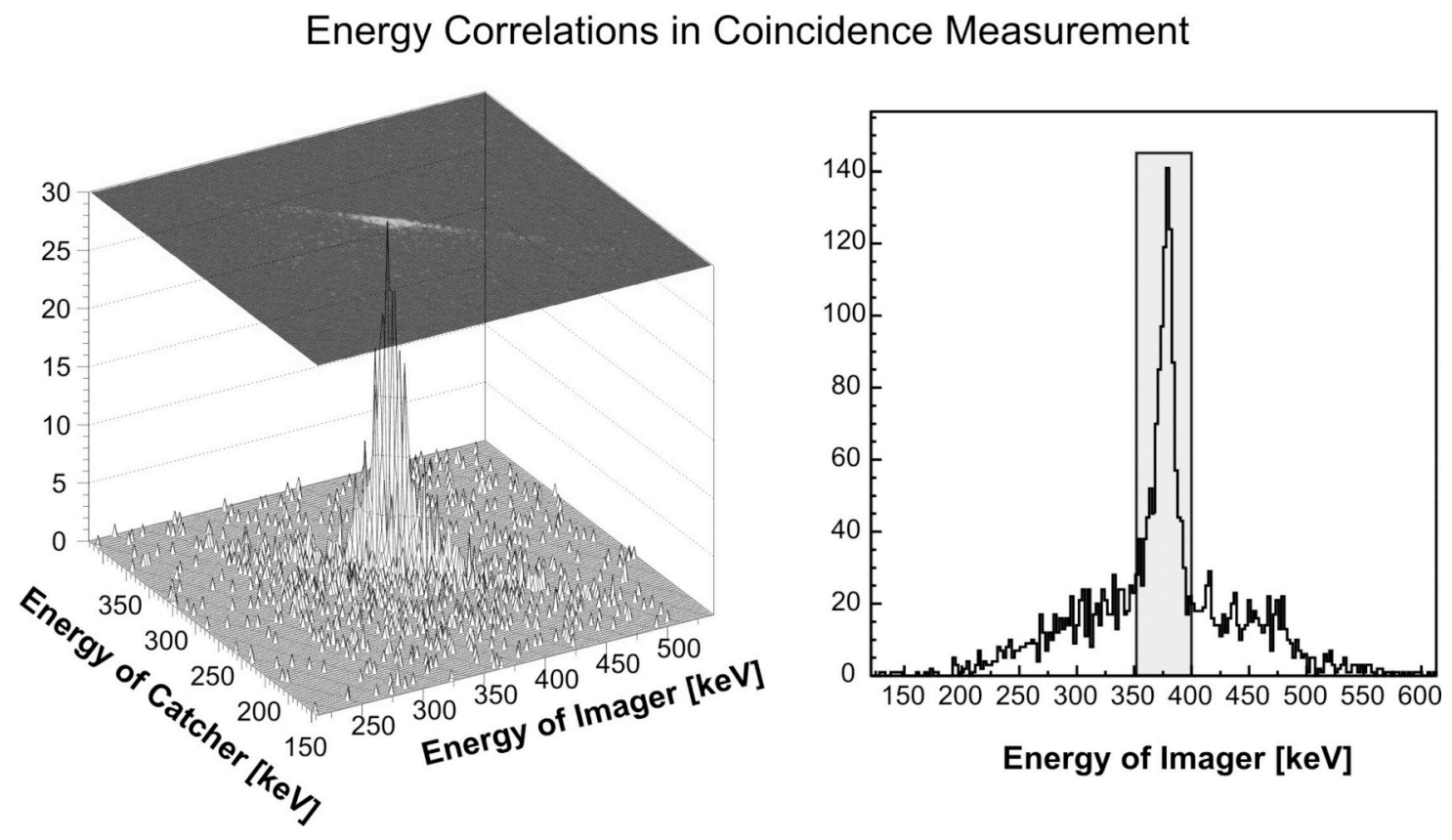

Fig. 5: Measured energy correlation between imager and catcher detector.
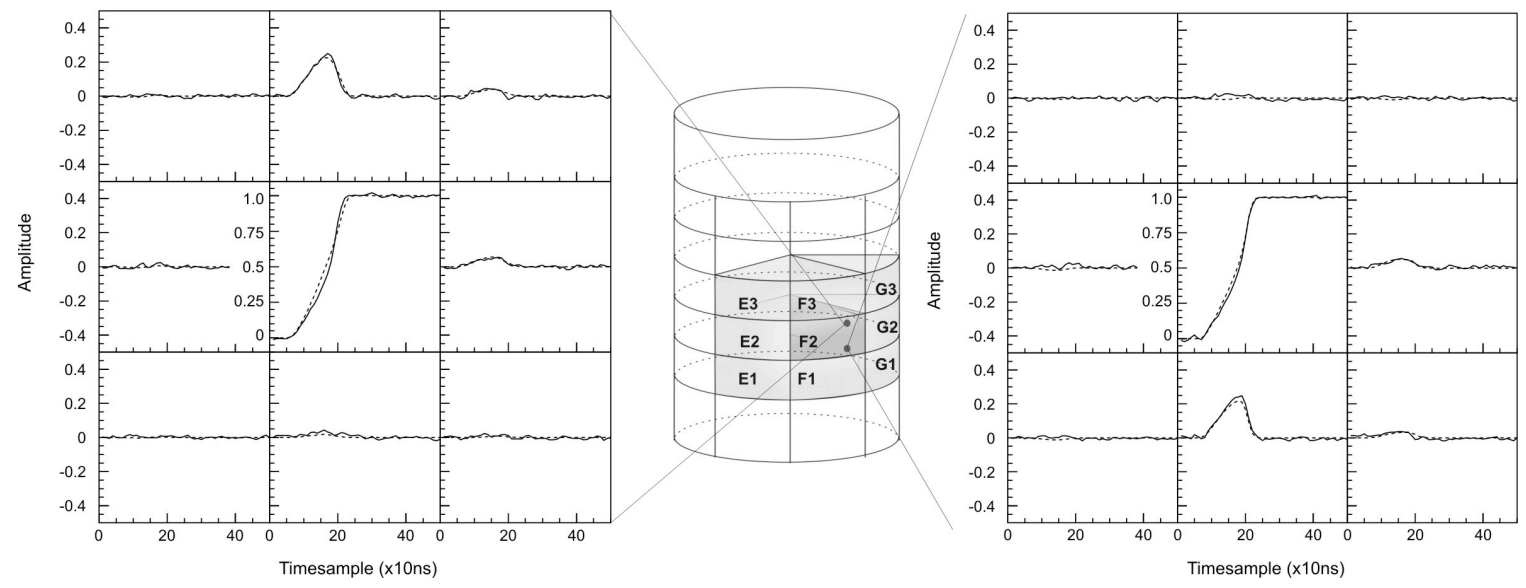

Fig. 6: Set of segment signals for two location indicated in the middle. The signals on the left reflect an interaction closer to the upper segment F3, the interaction on the right is closer to the lower segment F1. The solid lines are measured, the dashed lines calculated signals. 

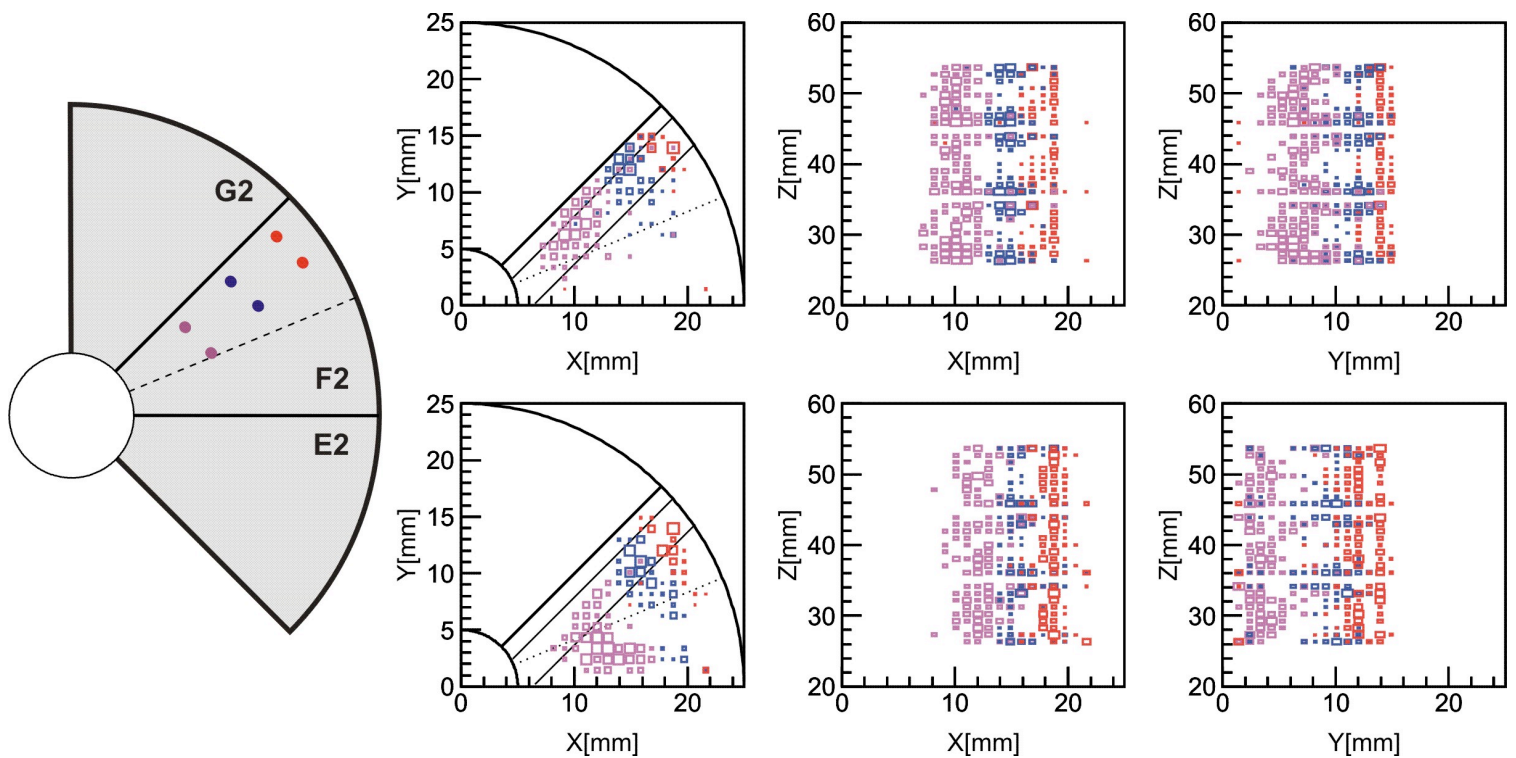

Fig. 7: Location of collimator positions (left) and corresponding deduced positions by signal decomposition calculations (right). The top row on the right reflects the positions on the line closer to segment G2.
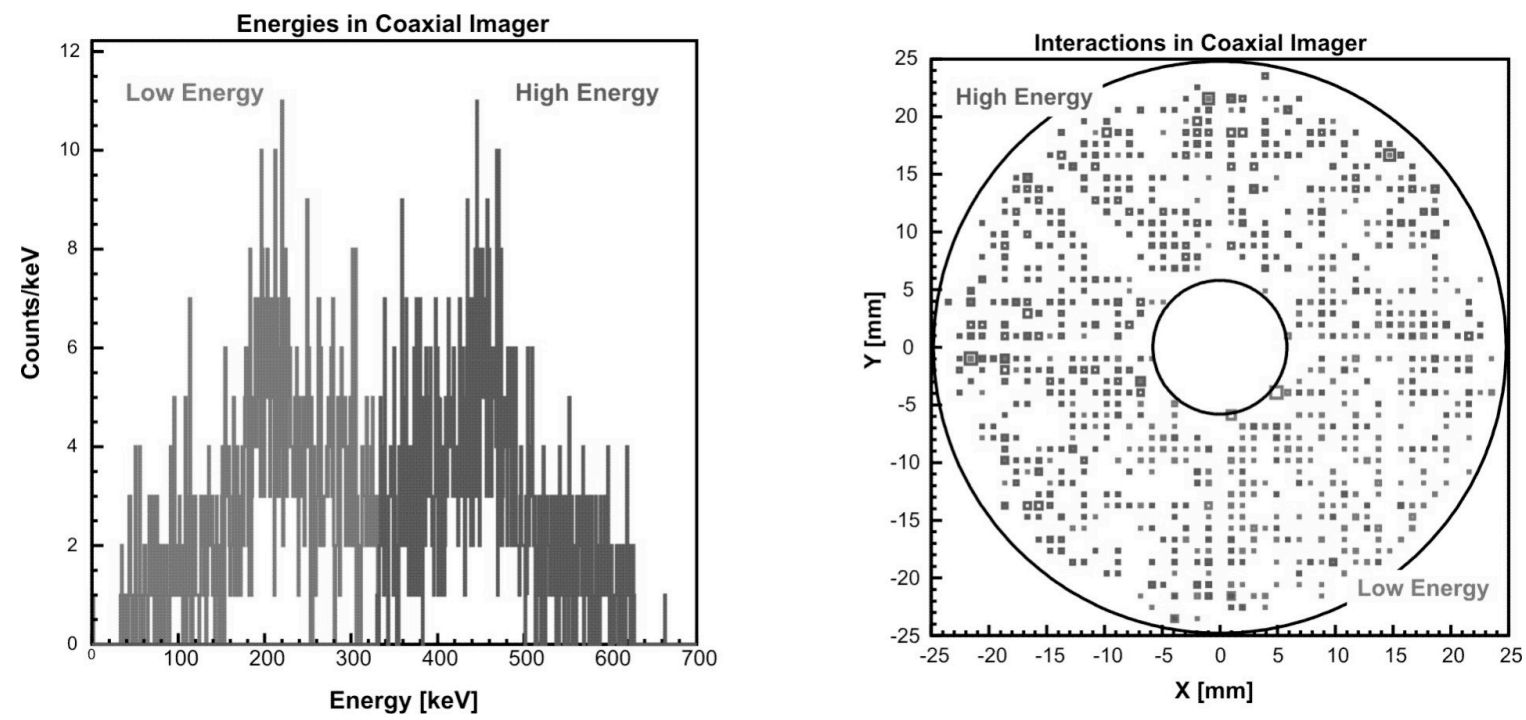

Fig. 8: Energies (left) and positions (right) identified and determined by signal decomposition calculations after illuminating the detector with a ${ }^{137} \mathrm{Cs}$ source as indicated on the right. The light boxes indicate the low energies and therefore the first of the two interactions. 



Fig. 9: Images deduced by the measured energies and positions of two interactions in the detector. The left image was determined with simple cone back projection, the image on the right was obtained with an iterative list-mode maximumlikelihood method. 